\title{
PROGRESS IN LARGE-SCALE FEMTOSECOND TIMING DISTRIBUTION AND RF-SYNCHRONIZATION
}

\author{
F. X. Kärtner, F. Ö. Ilday, J. Kim, A. Winter ${ }^{1}$, F. Grawert, H. Byun, and J. Chen, MIT, Cambridge, MA 02139, USA \\ ${ }^{1}$ also with Hamburg University and DESY, Hamburg, Germany
}

\section{Abstract}

Fourth-generation Light sources such as the European X-Ray free electron laser facility (XFEL), the Linear Coherent Light Source at SLAC (LCLS) as well as the ELLETRA-Facility at Trieste require femtosecond synchronization between low-level RF-systems, photoinjector laser, as well as potential probe lasers in these facilities. We recently proposed a modular system that is capable of achieving synchronization of various RF- and optical sub-systems with femtosecond precision over distances of a few kilometers [1], the typical size of the proposed facilities. In this paper, we report on recent progress towards the realization of such a system. Especially the construction of a low jitter optical master oscillator that generates the optical pulse stream that is necessary to stabilize the fiber transmission links of such a system and simultaneously carriers the timing information to the individual optical and RF sub-systems.

\section{INTRODUCTION}

The timing and synchronization system in the context of a seeded fourth-generation light source is depicted in Fig. 1. An optical master oscillator (OMO) in the form of a mode-locked laser is tightly synchronized to the reference microwave oscillator (RMO), which times the overall facility. Very low noise microwave oscillators that may serve as a reference microwave oscillator with a superior

$4^{\text {th }}$ Gen. Light Sources: XFEL

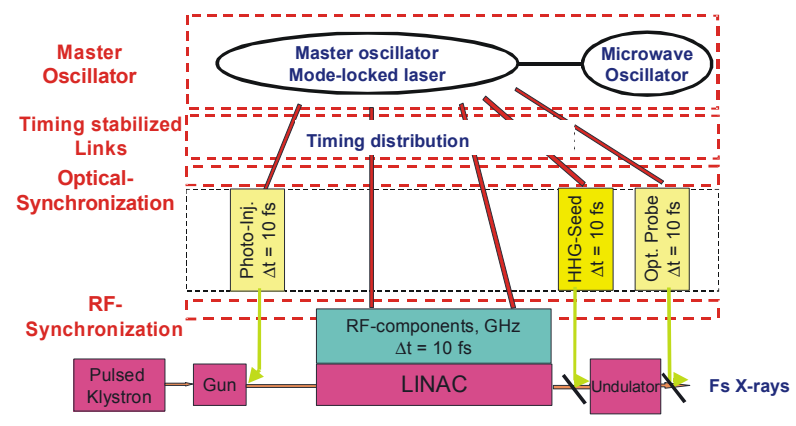

Fig. 1: Schematic outline of timing distribution and synchronization for FEL facility.

timing jitter well below $10 \mathrm{fs}$ within the typical repetition rate of the RF-macro pulses are commercially available, for example sapphire loaded dielectric resonator oscillators [2]. The OMO, i.e. mode-locked laser, generates a stream of very low jitter sub-picosecond

\footnotetext{
*Work supported by MIT, ONR, AFOSR and DESY
}

"kaertner@mit.edu pulses that are distributed to the individual sub-systems that need tight synchronization to the microwave reference of the facility. The optical pulse train has to fulfill two tasks. First, it serves as the means for stabilizing the group delay of the pulses in the fiber link to a multiple of the repetition time of the pulses with femtosecond precision. Second a fraction of the pulse train is coupled out from each end of the fiber links. The output pulse train at each fiber end can be used for synchronization of microwave or optical sub-systems to the RMO.

The use of an optical pulse train for timing distribution has many advantages compared to an interferometric stabilization of a fiber link. These are in particular:

- The RF-signal is encoded in the pulse repetition rate and any harmonic can be recovered directly at the fiber end.

- The group delay of the fiber link is directly stabilized rather than the phase delay. For a typical fiber link of $1 \mathrm{~km}$ in length group and phase delay may start to differ by more than 10 fs for $1^{\circ} \mathrm{C}$ change in temperature. In addition polarization mode dispersion is also on the order of $100 \mathrm{fs}$ times the square root of length in kilometers. Brillouin scattering and residual reflections in fiber discontinuities are strongly suppressed.

- Optical cross-correlation can be used both for link stabilization as well as local synchronization of additional optical sources.

- The pulse trains can be directly used to seed amplifiers at end stations.

These advantages also come at a prize. Sub-picosecond pulses, as necessary for femtosecond synchronization, strongly disperse in the fiber and therefore, dispersion compensated fiber links must finally be used and nonlinear effects must be accounted for.

It should be mentioned, that in principle the RF-signal and phase can also be encoded in the optical frequency and phase via a stabilized femtosecond laser frequency comb [3]. However, this would necessitate the implementation of such combs on both ends of each fiber link, which is an expensive solution.

In the following, we will discuss the requirements and hurdles to overcome in implementation of the proposed overall timing distribution system.

\section{RF-SYNCHRONIZATION}

RF synchronization essentially means to extract from the optical pulse stream leaving the fiber a highly stable and low jitter RF-signal either at the fundamental 
repetition rate of the pulse train or eventually one of its harmonics. Upon photo-detection, the short optical pulses, of sub-picosecond length, are converted into a train of electronic pulses of typically $50-100$ ps length if a $10 \mathrm{GHz}$ detector is used or even longer depending on the detectors bandwidth, see Fig. 2. The short optical pulses are

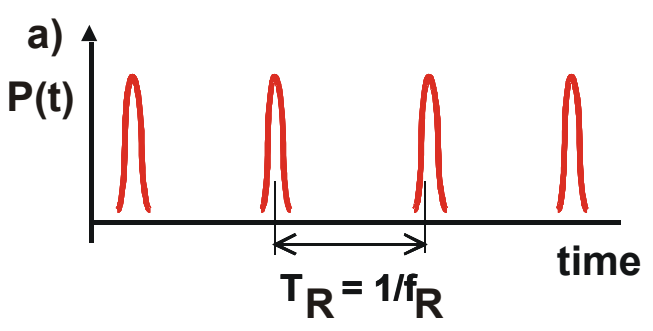

b)

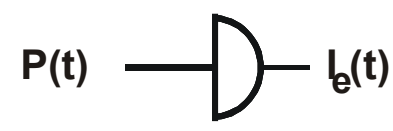

c)
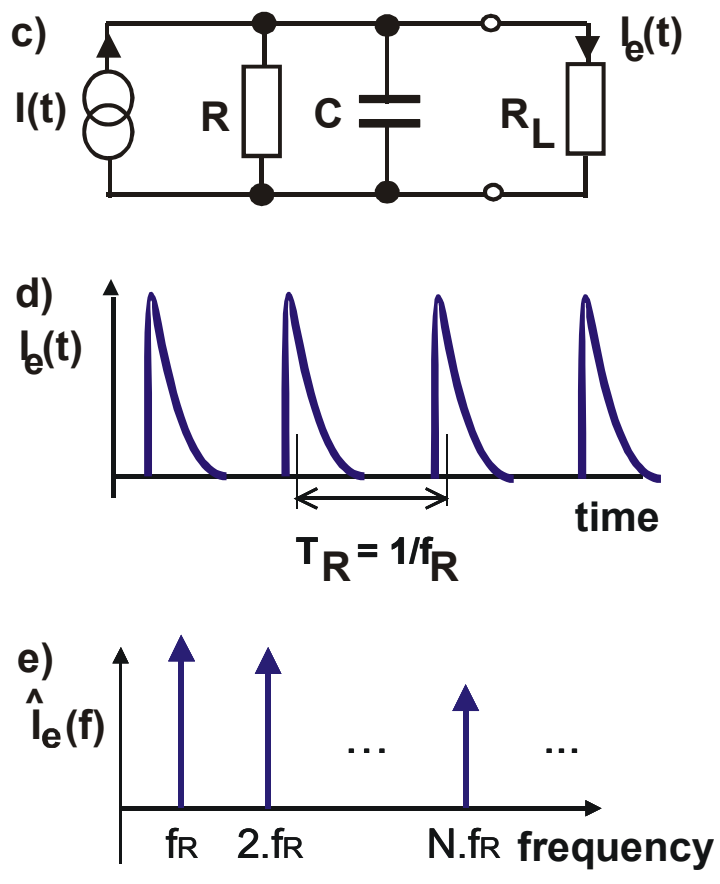

Fig. 2: a) Optical pulse train with instantaneous power $\mathrm{P}(\mathrm{t})$; b) Photo detection with external current $\mathrm{I}_{\mathrm{e}}(\mathrm{t})$; $\left.\mathrm{c}\right)$ Equivalent circuit of photo detector with external load; d) External current; e) Fourier components of external current.

eventually converted into more than a 1000 times longer electronic pulses $I_{e}(t)$ external to the photo detector. A reverse biased photo-detector can be modeled as a parallel $\mathrm{RC}$-circuit driven by an ideal current source following precisely the optical intensity profile, $\mathrm{I}(\mathrm{t}) \sim \mathrm{P}(\mathrm{t})$, see Figure $2 \mathrm{~b}$ and $2 \mathrm{c}$. The capacitor models the space-charge capacitance of the pn-junction. The output pulse stream of the photo-detector is the convolution of the optical pulse train with the internal photo-detector impulse response.
Or expressed differently, in the frequency domain all Fourier components $\hat{I}_{e}\left(\omega_{n}\right)$ of the periodic power variation in the optical pulse stream are multiplied by the frequency response of the photo-detector.

$$
H(\omega)=\frac{R_{L}}{R_{p}} \frac{1}{1-i \omega \tau}
$$

evaluated at each frequency component $\omega_{n}=2 \pi f_{n}=2 \pi n f_{r}$. Here, $R_{p}=\left(R / / R_{L}\right)$. Therefore, the different harmonics are phase shifted or experience a group delay

$$
\tau_{n}=-\left.\frac{d}{d \omega} \arg (H(\omega))\right|_{\omega_{n}}=\frac{\tau}{1+\left(\omega_{n} \tau\right)^{2}} .
$$

This formula essentially tells us that all harmonics within the detector bandwidth simply see the mostly constant group delay due to the RC-lowpass filter. However, the space charge capacitance of the detector depends on the incoming optical intensity or pulse energy. Therefore, intensity fluctuations are converted to timing fluctuations of the detector output signals via the AM-to-PM conversion coefficient

$$
C_{R F}^{A M-P M}=\left.\frac{d \tau}{d P}\right|_{P_{0}}=\left.\tau \frac{1}{C} \frac{d C}{d P}\right|_{P_{0}}
$$

This simple model explains already one cause that might lead to an intensity to phase conversion due to photo detection as has been observed recently $[4,5]$. Additional nonlinear effects during saturation of the diode in pulse operation may contribute to this effect and a more thorough investigation is necessary. We have measured this conversion coefficient with the setup shown in Figure 3 and results for $10 \mathrm{GHz}$ photo detectors from EOT are shown in the inset for a reverse bias voltage of $6 \mathrm{~V}$.

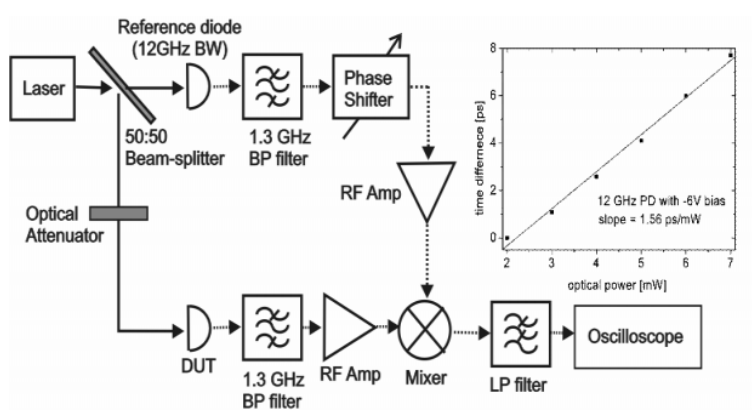

Fig. 3. AM-to-PM conversion factor measurement setup and the result when the reverse bias is set to $6 \mathrm{~V}$. The measured slope is $1.56 \mathrm{ps} / \mathrm{mW}$.

Typical numbers for the conversion coefficient may range from $C_{R F}^{A M-P M}=1-5 \mathrm{ps} / \mathrm{mW}$ depending on bias conditions, which significantly change the space charge capacitance and the sensitivity of the capacitance to power fluctuations, which are also consistent with conversion factors stated in [5]. To obtain an RF output signal with about $80 \mathrm{~dB}$ SNR in a $100 \mathrm{kHz}$ bandwidth an optical power of about $5 \mathrm{~mW}$ is necessary on the photo detector. Then even at a relative intensity noise level of $0.1 \%$ AM- 
to-PM conversion results in a timing jitter ranging from 525 fs, which clearly can not be neglected for synchronization on the $10 \mathrm{fs}$ level. Thus long term stable RF-extraction from a pulsed optical source by direct photo detection is only possible, if the diode power on the detector is actively stabilized using the DC-output of the detector and a proper feedback loop.

An alternative way to overcome the AM-to-PM excess noise has been recently demonstrated [6] by implementing a timing transfer in the optical domain before detection, see Fig. 4.

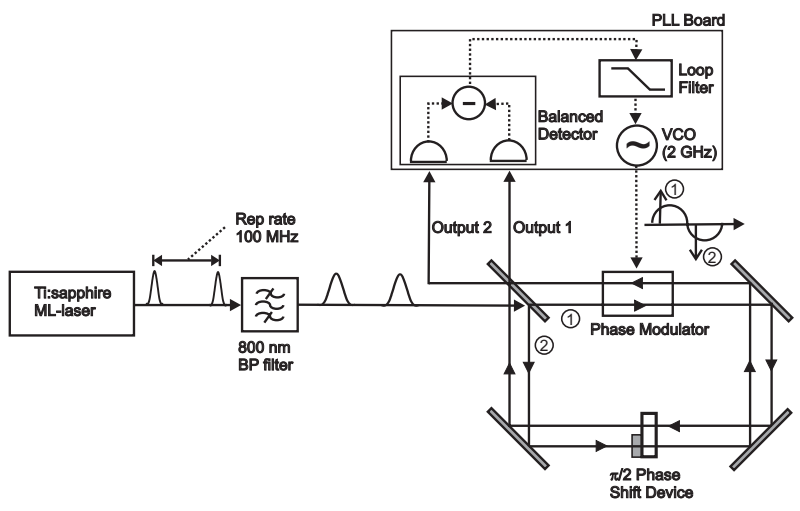

Fig. 4: Synchronization scheme for extraction of a $2 \mathrm{GHz}$ signal from a $100 \mathrm{MHz}$ repetition rate Ti:sapphire laser.

While still in the optical domain, the timing information is transferred into an intensity imbalance between two beams by sending the pulse train through a balanced sagnac interferometer. The phase modulator in the sagnac loop is positioned such that the counter clock-wise circulating pulse trains experience a differential phase shift, if they do not properly line up with the zero crossings of the phase modulator driven by the VCO running at the $20^{\text {th }}$ harmonic of the pulse repetition rate, that are $2 \mathrm{GHz}$ and $100 \mathrm{MHz}$, respectively. For more details see Ref.[6].

\section{TIMING STABILIZED FIBER LINKS}

According to Fig. 1, the pulse stream from the OMO will be distributed over timing stabilized fiber links. Such a link may be constructed as shown in Fig. 5. Precise transfer of RF-signals through fiber links has been demonstrated recently $[7,8]$ using cw-lasers and even more recently using pulse trains from mode-locked lasers to about $50 \mathrm{fs}$ precision [9] within a bandwdith of $100 \mathrm{kHz}$. The latter result was achieved using RF-locks only. We have demonstrated previously [10], that a balanced crosscorrelator enables sub-femtosecond synchronization between different laser sources. We envision that an optical cross-correlator can be used to further stabilize the fiber link to the femtosecond level after pre-stabilization of the link to the $100 \mathrm{fs}$ level using RF-locks. First results towards such a timing stabilized fiber link are presented in these proceedings in Ref. [11].
If the fiber length is $\mathrm{L}$, and we assume that no length fluctuations are faster than $(2 \mathrm{~nL}) / \mathrm{c}$, where $\mathrm{n}$ is the refractive index of the fiber and $\mathrm{c}$ is the vacuum velocity of light, it is in principle possible to take out all fiber fluctuations by the feedback loop and the pulse train is ideally transmitted over the fiber without additional timing jitter.

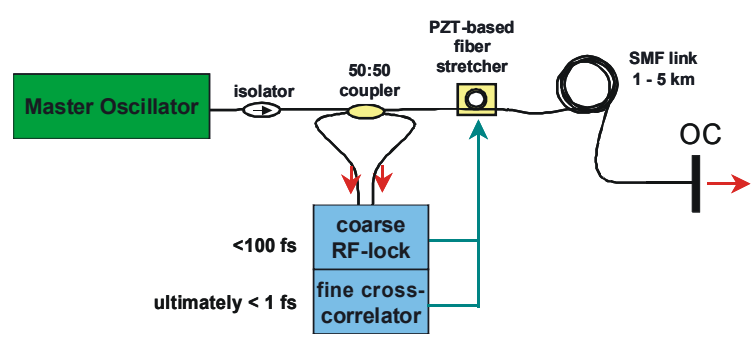

Fig. 5: Schematic of a timing stabilized fiber link. The coarse stabilization is accomplished by an RF-lock, whereas the fine adjustment will be accomplished by optical cross-correlation.

\section{OPTICAL MASTER OSCILLATOR}

The most promising candidates for ultra-low jitter optical master oscillators are Er/Yb-glass lasers [12], passively mode-locked Er-doped fiber lasers [13] and Yb-doped fiber lasers [14]. Er/Yb-glass lasers with sub-20 fs timing jitter have been demonstrated recently [12]. The crucial performance indicator for such a source is the phase noise or timing jitter integrated from $\mathrm{c} /(2 \mathrm{~nL})$ to the Nyquist frequency, i.e. half the repetition rate of the laser. This jitter will set an inherent limitation to the precision with which the pulse train can be distributed in the facility. Note, this is not necessarily a limitation for the RFdistribution, if the flywheel effect of the VCO in the RFrecovery according to Fig. 4 is properly exploited. In the final application, the OMO will follow within a certain bandwidth $f_{O M O}$ tightly the RMO. If the bandwidth of the fiber link stabilization $f_{\text {link }}$ is greater than $f_{O M O}$ and the bandwith of the lock for the RF extraction $f_{R F}$ is less than $f_{O M O}$, than the phase noise of the finally extracted RF is identical to the phase noise of the RMO for frequencies less than $f_{R F}$ and identical to the phase noise of the VCO for frequencies beyond $f_{R F}$. Then even high frequency fiber length fluctuations outside of $f_{R F}$ are suppressed. Nevertheless, a very low jitter OMO is indispensable for femtosecond timing distribution. We have constructed stably operating sub-picosecond Yb-fiber, Er-fiber and bulk Er/Yb-glass lasers and evaluated the timing jitter of the different sources. Figure 6 (a) and (b) show the typical setup of the fiber lasers and Er/Yb-glass bulk lasers. The fiber lasers are mode-locked by nolinear polarization rotation and operate in the stretched pulse mode [15]. Due to the minimum length required for strong polarization rotation and the other fiber components, the repetition rate of fiber lasers is at maximum on the order of a few hundred $\mathrm{MHz}$. In contrast the Er/Yb-glass bulk laser is mode-locked by a seminconductor saturable absorber 
[16]. The length of the gain medium can be as short as a few millimeter and, therefore, if necessary these lasers can be scaled to tenth of $\mathrm{GHz}$ in repetition rate [17].

We implemented both an all fiber Er-fiber laser, Yb-fiber laser where dispersion compensation was achieved with an intracavity grating pair and an Er/Yb-glass bulk laser. More details on the operating principles of the fiber lasers can be found in Ref. [18] and references therein.
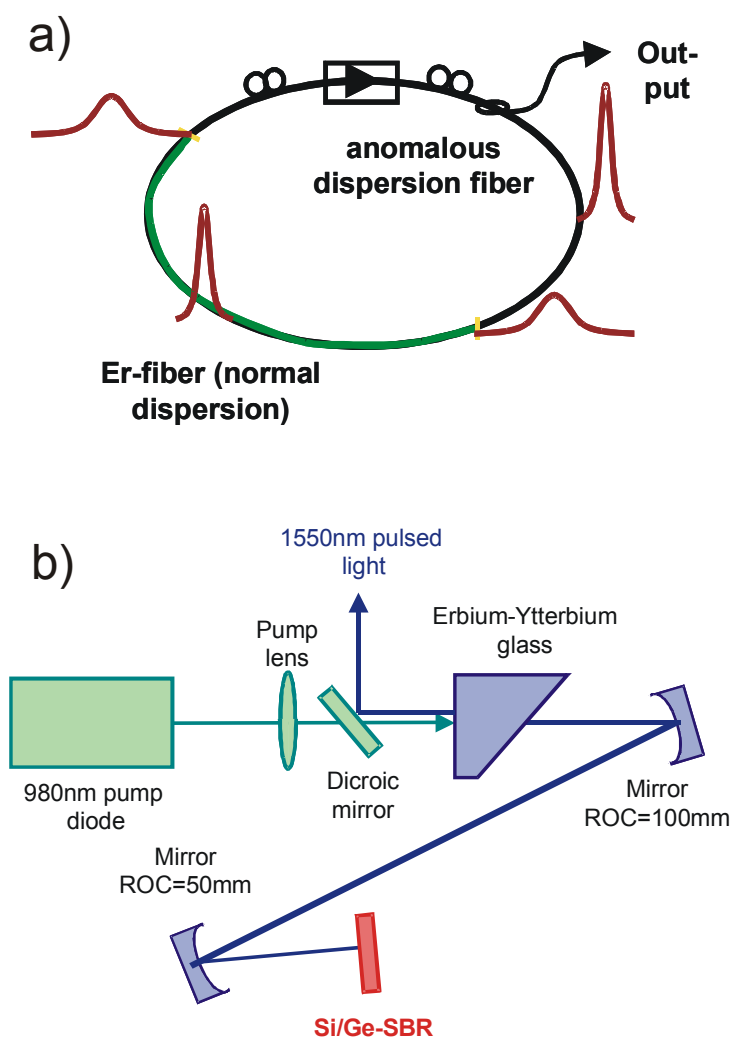

Fig. 6: Schematic of (a) a stretched pulse Er-fiber laser mode-locked by nonlinear polarization rotation and (b) a $\mathrm{Er} / \mathrm{Yb}$-glass laser mode-locked by a semiconductor saturable absorber mirror.

Fig. 7 shows the Single-Sideband (SSB) Phase Noise of an $\mathrm{Yb}$-fiber laser, Er-fiber laser and $\mathrm{Er} / \mathrm{Yb}$-glass laser measured with an Agilent 5052a signal source analyzer. The fiber lasers had a repetition rate of $36 \mathrm{MHz}$ and the $\mathrm{Er} / \mathrm{Yb}$-glass laser of $216 \mathrm{MHz}$. The measurements were carried out on the corresponding harmonic at $1.3 \mathrm{GHz}$. The integrated timing jitter from $10 \mathrm{kHz}$ to $18 \mathrm{MHz}$, i.e. half the repetition rate of the fiber lasers is found to be 18 fs, 48 fs and 38 fs respectively. The value for the Yb-fiber laser is a record low number for the jitter of mode-locked lasers and can be well understood by the established theories on noise in mode-locked laser. This stretched pulse mode-locked $\mathrm{Yb}$-fiber laser generates the shortest pulses and operates close to the zero average dispersion point of the cavity. In that case, frequency fluctuations can not couple to timing fluctuations, which is known as the Gordon-Haus jitter. The timing jitter also scales proportional to the pulse width of the generated pulses.
Both facts contribute to the low timing jitter of this $\mathrm{Yb}$ laser $[19,20]$. This operation principle will be also applied in the next version of the Er-fiber laser. In contrast, in the $\mathrm{Er} / \mathrm{Yb}$-glass bulk laser the intracavity dispersion is already very small from the beginning and does not play any significant role in the pulse shaping and timing jitter dynamics, however, it produces longer pulse in the range of 100-200fs. Also the relative intensity noise (RIN) of the fiber lasers has been measured, see Ref[18]. The integrated RIN measured from $10 \mathrm{kHz}$ to $1 \mathrm{MHz}$ is about $0.04 \% \mathrm{rms}$ for the YDFL, and $0.03 \% \mathrm{rms}$ for the EDFL. With a typical power level of $10 \mathrm{~mW}$ on the photo detector the AM-to-PM conversion discussed in section III predicts already a measurement related timing jitter from AM-to-PM conversion effects of about $5 \mathrm{fs}$. This is already a considerable fraction of the $18 \mathrm{fs}$ timing jitter measured in the case of the Yb-fiber laser. Thus a more detailed analysis of measurement related and actual laser related noise has to be undertaken in the future.

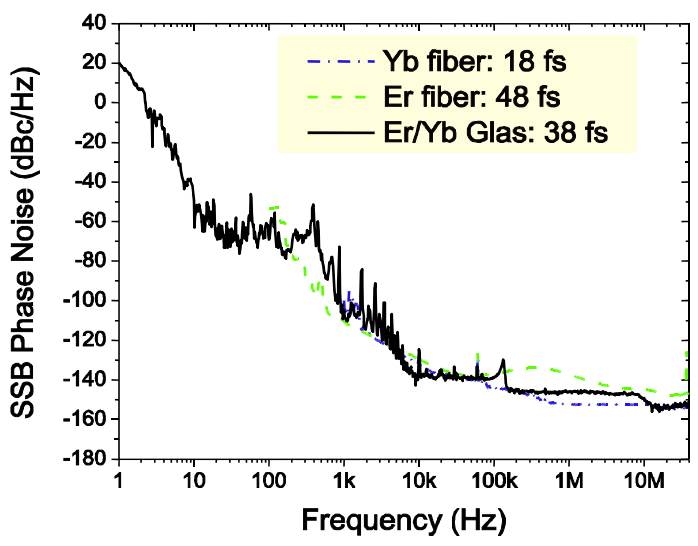

Fig. 7: Single-Sideband Phase Noise of an Yb-fiber laser, Er-fiber laser and $\mathrm{Er} / \mathrm{Yb}$-glass laser. The integrated timing jitter from $10 \mathrm{kHz}$ to $18 \mathrm{MHz}$, i.e. half the repetition rate of the fiber lasers is stated explicitly.

The use of fiber lasers is a natural choice for implementing an optical master oscillator, because of the ease of coupling to the fiber distribution system, their good long-term stability, and the well-developed and mature components. However, should higher repetition rate lasers become necessary for optimum timing distribution, the properly packaged Er/Yb-glass lasers can be made equally stable.

\section{OPTICAL-TO-OPTICAL SYNCHRONIZATION}

Synchronization is necessary not only between optical and RF-subsystems but also between different optical systems, for example, the photo-injector laser and the master oscillator as shown in Fig. 1. Figure 8 shows how the optical-to-RF synchronization scheme based on timing transfer in the optical domain, discussed in section II, can be used to tightly synchronize two pulsed lasers with each other. By using the RF-synchronization module first the 
RF signal is extracted from one laser (ML-laser 1 in Fig. 8). This RF-signal drives the phase modulator of another RF-synchronization module. However, the error signal from balanced detector of the second loop drives a piezoelectric transducer (PZT) that controls the repetition rate of the second laser (ML-laser 2 in Fig. 8) rather than the VCO. In this way, an effective synchronization of multiple lasers can be achieved.

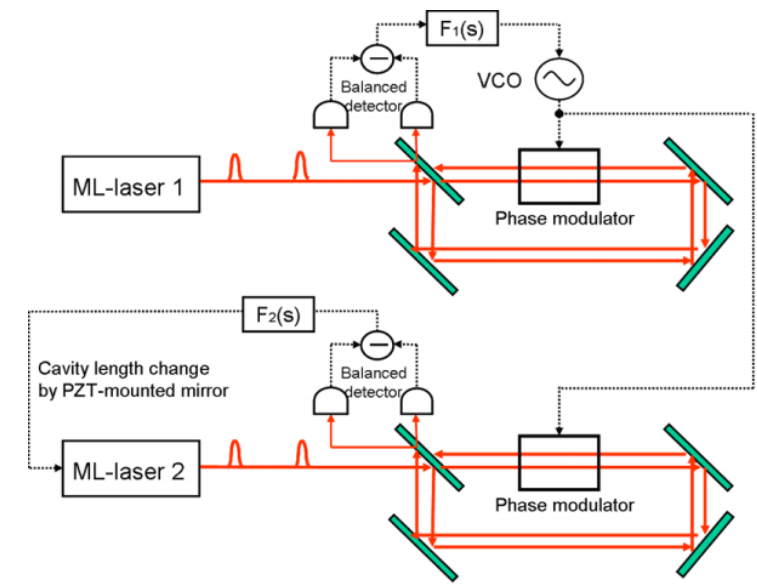

Fig. 8: Optical-to-optical synchronization scheme with the based on RF-synchronization techniques.

Another method for optical-to-optical synchronization is based on balanced optical cross correlation. This method enabled so far the most accurate synchronization of multiple laser sources [10].

\section{CONCLUSION AND OUTLOOK}

In summary, we introduce a scalable timing distribution and synchronization scheme for future accelerator facilities and $4^{\text {th }}$ generation light sources which should finally enable synchronization on the $10 \mathrm{fs}$ level. We discussed in detail the necessary sub-systems to be developed. Major progress in the identification and construction of low jitter mode-locked lasers has been reported. Several laser sources with timing jitter levels below $50 \mathrm{fs}$ and an Yb-fiber laser with less than $18 \mathrm{fs}$ timing jitter have been realized which can be readily used for construction timing distribution system enabling sub$100 \mathrm{fs}$ timing jitter. The limiting aspects of AM-to-PM conversion that occurs in photo detection has been characterized and its impact in timing jitter measurements and synchronization was discussed.

\section{REFERENCES}

[1] J. Kim, F. Ö. Ilday, F. X. Kärtner, O. D. Mücke, M. H. Perrott, W. H. Graves, D. E. Moncton and T. Zwart, "Large Scale Timing Distribution and RFSynchronization for FEL-Facilities," FELConference 2004, Trieste, Italy, Aug. 26-29, 2004.

[2] Poseidon Scientific, http://www.psi.com.au.

[3] R. Holzwarth, Menlo-Systems, Priv. Comm..
[4] E. N. Ivanov, S. A. Diddams and L. Hollberg, IEEE J. Sel. Top. Quant. Elec. 9, 1059 (2003).

[5] A. Bartels, S. A. Diddams, C. W. Oates, G. Wilpers, J. C. Bergquist, W. H. Oskay, and L. Hollberg, Opt. Lett. 30, 667 (2005).

[6] J. Kim, F. X. Kärtner, and M. H. Perrott, Opt. Lett. 29, 2076 (2004).

[7] J. Ye, J.-L. Peng, R. J. Jones, K. W. Holman, J. L. Hall, D. J. Jones, S. A. Diddams, J. Kitching, S. Bize, J. C. Bergquist, L. W. Hollberg, L. Robertsson, L.-S. Ma, J. Opt. Soc. Am. B 20, 1459 (2003).

[8] K. W. Holman, D. J. Jones, D. D. Hudson, J. Ye, Opt. Lett. 29, 1554 (2004).

[9] K. W. Holman, D. D. Hudson, D. J. Jones, J. Ye, Opt. Lett. 30, 1225 (2005).

[10] T. R. Schibli, J. Kim, O. Kuzucu, J. T. Gopinath, S. N. Tandon, G. S. Petrich, L. A. Kolodziejski, J. G. Fujimoto, E. P. Ippen, F. X. Kärtner, Opt. Lett. 28, 947 (2003).

[11] A. Winter, H. Schlarb, B. Schmidt, F. O. Ilday, J. W. Kim, J. Chen, G. Grawert, and F. X. Kärtner, "Stabilized Optical Fiber Links for the XFEL," In Proceedings of PAC 2005.

[12] J. B. Schlager, B. E. Callicoatt, R. P. Mirin, N. A. Sanford, D. J. Jones, J. Ye, Opt. Lett. 28, 2411 (2003).

[13] G. Lenz, K. Tamura, H. A. Haus, E. P. Ippen, Opt. Lett. 20, 1289 (1995).

[14] F. Ö. Ilday, J. R. Buckley, H. Lim, F. W. Wise, W. G. Clark, Opt. Lett. 28, 1365 (2003).

[15] K. Tamura, E. P. Ippen, H. A. Haus, and L. E. Nelson, Opt. Lett. 18, 1080-1082 (1993).

[16] F. J. Grawert, S. Akiyama, J. T. Gopinath, F. Ö. Ilday, H. Shen, J. Liu, K. Wada, L. C. Kimerling, E. P. Ippen and F. X. Kärtner, Opt. Lett. 30, 329-331 (2005).

[17] S. C. Zeller, L. Krainer, G. J. Spuehler, R. Paschotta, M. Golling, D. Ebling, K. J. Weingarten, and U. Keller, 30th European Conference on Optical Communication. ECOC 2004, 2004, pt. 4, p 918-19 vol.4 “

[18] A. Winter, P. Schmueser, H. Schlarb, B. Schmidt, F. O. Ilday, J. W. Kim, J. Chen, and F. X. Kärtner, "Phase Noise Characteristics of Fiber Lasers as Potential Ultra-Stable Master Oscillators," In Proceedings of PAC 2005.

[19] S. Namiki, and H. A. Haus, IEEE J. Quantum Electron. 33, 649-659 (1997).

[20] C. X. Yu, S. Namiki, and H. A. Haus, IEEE J. Quantum. Electron. 33, 660-668 (1997). 